Table A, S1 file

\title{
Characteristics of $\delta^{15} \mathrm{~N}$ values and coagulation parameters from samples analyzed in this study.
}

Variables were natural-log transformed to improve the normality of the frequency distributions. Skewness and kurtosis values are given for data before and after natural-log transformation of variables. For simplicity, only natural-log transformed dependent (coagulation) variables were used in regression analysis. $\delta^{15} \mathrm{~N}$ was used as the independent variable and was not transformed. A variable with symmetrical distribution has a skewness of zero. The kurtosis for a standard normal distribution is three.

\begin{tabular}{|c|c|c|c|c|c|}
\hline & \multicolumn{5}{|c|}{ Untransformed Values } \\
\hline Variable & Mean \pm S.D. & Median (IQR) & Range & Skewness & Kurtosis \\
\hline$\delta^{15} \mathrm{~N}$ value $(\%)$ & $8.7 \pm 1.3$ & $8.4(1.58)$ & $6.1-14.5$ & 1.06 & 4.26 \\
\hline sP-selectin (ng/mL) & $34.6 \pm 11.4$ & $33.2(14.3)$ & $11.3-80.4$ & 0.72 & 3.62 \\
\hline Clotting factor II (\%) & $107.4 \pm 20.1$ & $103.0(22.0)$ & $35.0-180.0$ & 0.80 & 4.01 \\
\hline Clotting factor V $(\%)$ & $81.6 \pm 32.0$ & $87.0(38.0)$ & $3.5-211.0$ & -0.47 & 3.05 \\
\hline Fibrinogen $(\mathrm{ng} / \mathrm{mL})$ & $345 \pm 106$ & $333.0(124.0)$ & $142-842$ & 0.70 & 4.37 \\
\hline PT (sec) & $12.7 \pm 1.61$ & $12.7(1.40)$ & $8.0-25.0$ & 1.91 & 14.2 \\
\hline INR & $1.01 \pm 0.17$ & $1.00(0.200)$ & $0.6-2.5$ & 2.47 & 19.3 \\
\hline \multirow[t]{2}{*}{ PTT (sec) } & $33.8 \pm 10.4$ & $32.0(6.00)$ & 14-199 & 9.88 & 148.1 \\
\hline & \multicolumn{5}{|c|}{ Natural Log-Transformed Values } \\
\hline Variable & Mean \pm S.D. & Median (IQR) & Range & Skewness & Kurtosis \\
\hline$\delta^{15} \mathrm{~N}$ value (\%o) & $2.16 \pm 0.14$ & $2.13(0.18)$ & $1.81-2.67$ & 0.66 & 3.28 \\
\hline sP-selectin (ng/mL) & $3.49 \pm 0.33$ & $3.50(0.43)$ & $2.43-4.39$ & -0.27 & 3.19 \\
\hline Clotting factor II (\%) & $4.66 \pm 0.18$ & $4.63(0.21)$ & $3.55-5.19$ & -0.11 & 5.70 \\
\hline Clotting factor V $(\%)$ & $4.27 \pm 0.60$ & $4.47(0.46)$ & $1.25-5.35$ & -1.87 & 6.53 \\
\hline Fibrinogen $(\mathrm{ng} / \mathrm{mL})$ & $5.79 \pm 0.32$ & $5.81(0.37)$ & 4.84-6.74 & -0.40 & 3.44 \\
\hline PT (sec) & $2.54 \pm 0.12$ & $2.54(0.11)$ & $2.19-3.46$ & 0.64 & 7.31 \\
\hline INR & $-4.02 \times 10^{-4} \pm 0.15$ & $0.00(0.20)$ & $-0.51-0.92$ & 0.69 & 7.22 \\
\hline PTT (sec) & $3.50 \pm 0.20$ & $3.47(0.18)$ & $2.64-5.29$ & 2.23 & 21.2 \\
\hline
\end{tabular}


Table B, S1 file

Demographics for the participant group that provided samples for PIVKA-II analysis.

\begin{tabular}{c|c|c|c|c|c}
\hline Group & $\begin{array}{c}\text { Sample } \\
\text { Size }\end{array}$ & $\begin{array}{c}\text { Age } \\
\text { (years) }\end{array}$ & $\begin{array}{c}\text { Age } \\
\text { Range }\end{array}$ & $\begin{array}{c}\% \\
\text { Female }\end{array}$ & $\begin{array}{c}\% \\
\text { Coastal }\end{array}$ \\
\hline All & 682 & $36.2 \pm 17.8$ & $14-79$ & 47.2 & 52.8 \\
\hline$C Y P 4 F 2 * 3$ & \multicolumn{5}{|l}{} \\
\hline$* 1 / * 1$ & 185 & $33.6 \pm 17.6$ & $14-79$ & 48.6 & 63.2 \\
\hline$* 1 / * 3$ & 329 & $37.1 \pm 17.8$ & $14-77$ & 47.1 & 54.7 \\
\hline$* 3 / * 3$ & 168 & $37.6 \pm 17.8$ & $14-79$ & 45.8 & 37.5 \\
\hline$G G C X$ R325Q & \multicolumn{5}{|l}{} \\
\hline CC & 164 & $35.3 \pm 17.2$ & $14-77$ & 53.7 & 53.7 \\
\hline $\mathrm{CT}$ & 354 & $36.7 \pm 18.1$ & $14-78$ & 47.2 & 50.3 \\
\hline $\mathrm{TT}$ & 164 & $36.2 \pm 17.8$ & $14-79$ & 40.9 & 57.3 \\
\hline VKORC1 $1173 A>G$ & \multicolumn{5}{|l}{} \\
\hline $\mathrm{AA}$ & 433 & $35.9 \pm 17.9$ & $14-79$ & 47.8 & 58.0 \\
\hline $\mathrm{AG}$ & 199 & $36.8 \pm 17.5$ & $14-79$ & 46.2 & 43.7 \\
\hline $\mathrm{GG}$ & 50 & $37.4 \pm 18.1$ & $14-77$ & 46.0 & 44.0 \\
\hline \multicolumn{6}{|l|}{}
\end{tabular}




\section{Fig A, S1 file}

\section{Sex differences in $\delta^{15} \mathrm{~N}$ values among Yup'ik participants.}

Females had higher $\delta^{15} \mathrm{~N}$ values compared to males $(\mathrm{P}<0.001)$. Median (IQR) $\delta^{15} \mathrm{~N}$ for males was 8.2 (1.3) and for females was 8.8 (1.7).

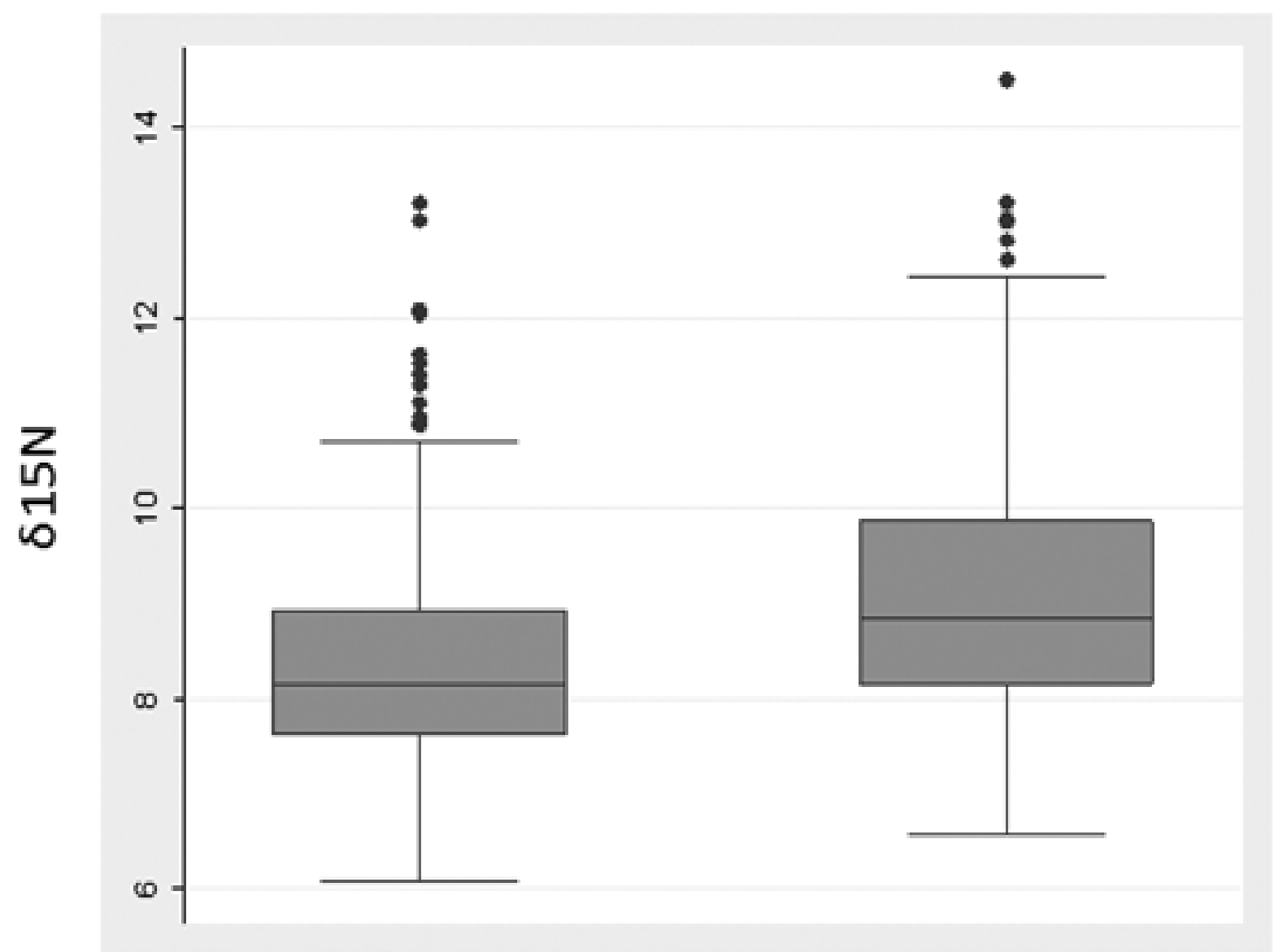

Male

\section{Female}


Fig B, S1 file

Distribution of $\delta^{15} \mathrm{~N}$ values from study participants stratified by; A) coastal and B) inland communities.

Coastal communities generally exhibited higher $\delta^{15} \mathrm{~N}$ values (mean \pm S.D., 9.1 \pm 1.5 ) than inland communities ( $8.4 \pm 0.9) ; \mathrm{P}<0.001$.

A) Coastal

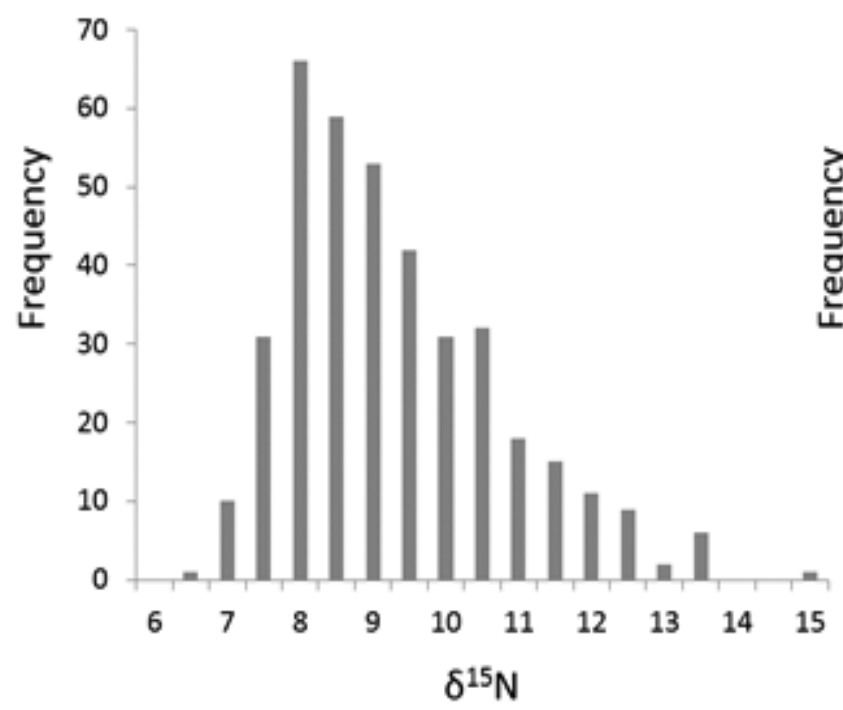

B) Inland

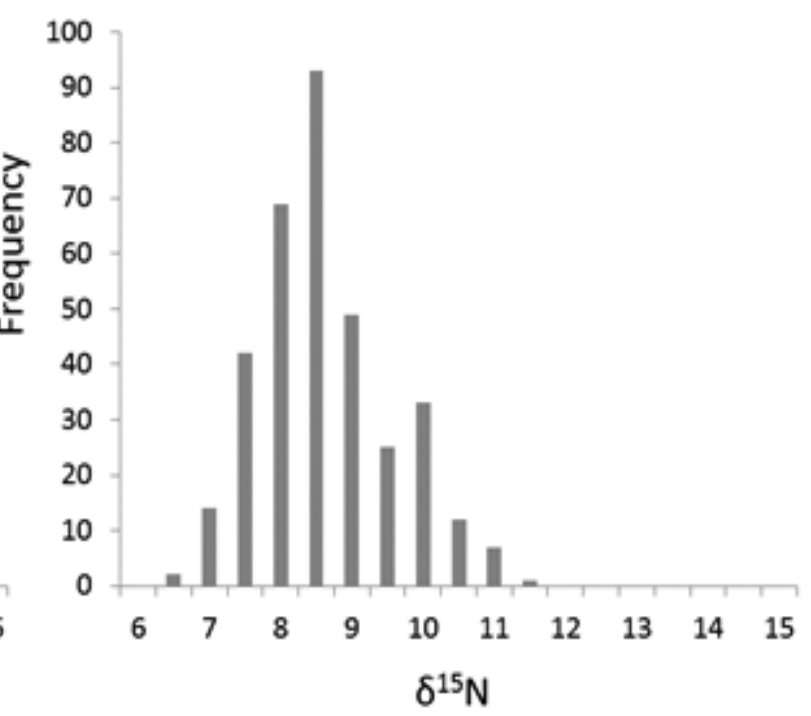




\section{Fig C, S1 file}

Effect of CYP4F2 genotype on plasma vitamin K levels.

Box-and-whisker plots comparing medians and IQRs of plasma VK1 concentrations for CYP4F2*3. Statistical data are summarized in Table 3. Using a two-sided t-test for comparing natural-log transformed plasma VK1 values, $\mathrm{P}=0.005$ for $* 3 / * 3$ vs. $* 1 / * 1$, $\mathrm{p}=\mathrm{P} .685$ for $* 1 / * 3$ vs. ${ }^{*} 1 / * 1$.

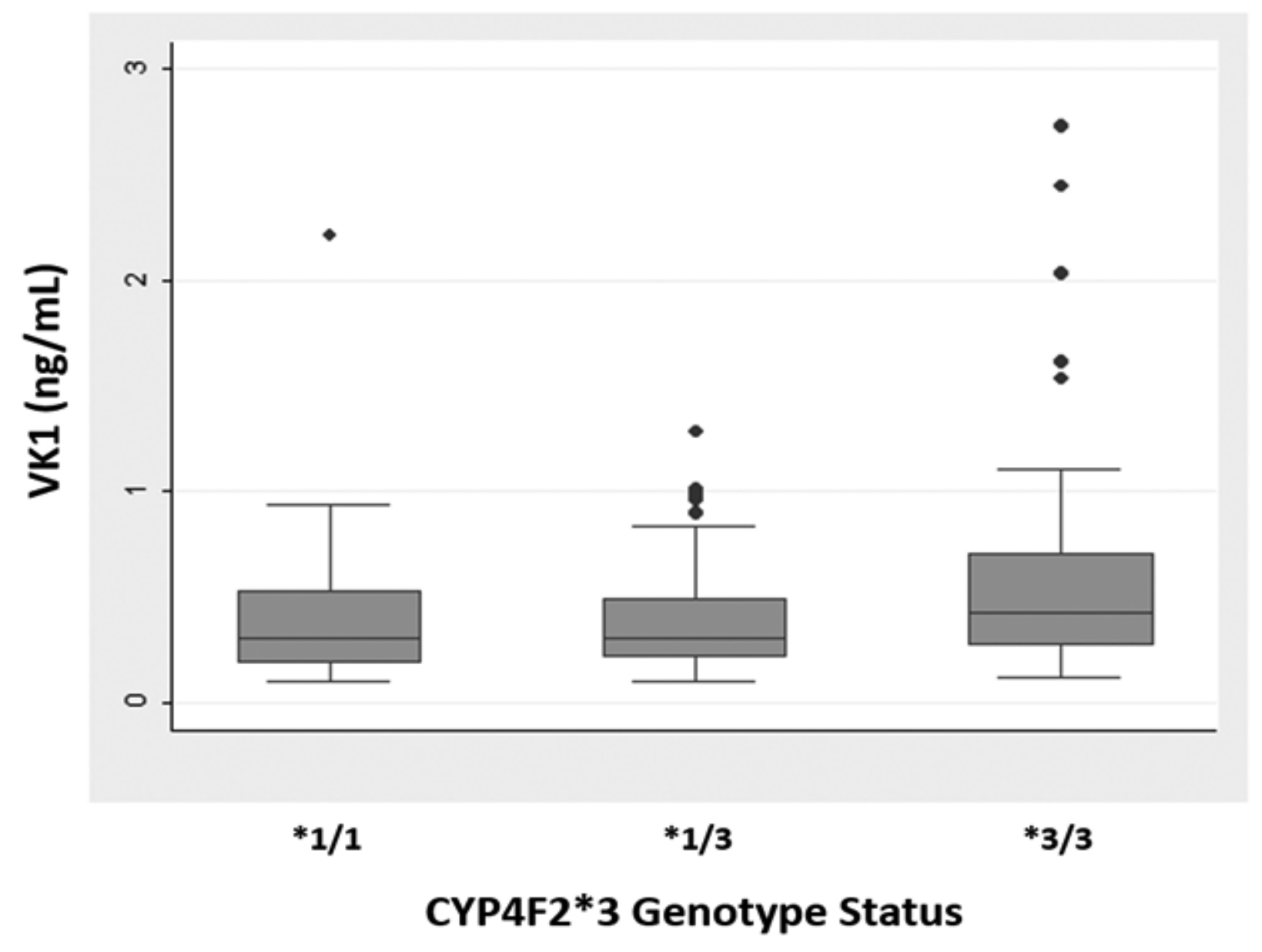

